\title{
Los recursos multimedia de refuerzo para el aprendizaje de herramientas informáticas, ¿cuál es su uso y valoración por parte del alumnado?
}

\author{
Pilar Bosch Roig ${ }^{a}$, Melani Lleonart García ${ }^{\mathrm{b}}$ y Jose Madrid García ${ }^{\mathrm{c}}$
}

${ }^{a}$ Dpto. de Conservación y Restauración de Bienes Culturales, Universitat Politècnica de València (mabosroi@upvnet.upv.es), ${ }^{b}$ Dpto. de Pintura, Universitat Politècnica de València (mellgar@upv.es) y ${ }^{\mathrm{c}}$ Dpto. de Conservación y Restauración de Bienes Culturales, Universitat Politècnica de València (jmadrid@crbc.upv.es).

\section{Abstract}

Many times, we have asked ourselves if the multimedia resources that we use in our subjects are really the most appropriate. Resources that involve an important teacher's work on preparing them. But, do they really fulfill or achieve what is being pursued? are they used or involve more problems than solutions? These may be some of the questions we can ask ourselves once the task is done. In the third year subject "Photography and documentation applied to the study of cultural heritage" of the Bachelor's Degree in Conservation and Restoration of Cultural Heritage from the Faculty of fine arts of the Universitat Politècnica de València (UPV), we have decided to do a quick control test in order to determine some variables which can help to work better these contents. In this subject, we have the opportunity to work in parallel with two groups of students who have been provided with a battery of different online resources. Through a survey applied to both groups, we have collected their used and valuation of the available multimedia resources to support the learning of computer tools needed for the development of the exercises and tasks assigned in this subject.

Keywords: learning resources, autonomous learning, evaluation, rubric, ICT, online resources.

\section{Resumen}

Muchas veces nos hemos podido preguntar si de verdad los recursos multimedia que empleamos en nuestras asignaturas son los más adecuados. Recursos que implican una importante labor de preparación por parte del profesorado. Pero ¿de verdad cumplen, o alcanzan, lo que se persigue?, ¿son utilizados?, o ¿implican más problemas, que soluciones?, pueden ser algunas de las preguntas que nos podemos hacer una vez terminada la tarea. En la asignatura "Fotografia y Documentación Aplicada al Estudio de los Bienes Culturales» de tercero de Grado en Conservación y Restauración de Bienes Culturales (Facultad de Bellas Artes, Universitat Politècnica de València), hemos decidido hacer una rápida prueba de control para poder determinar algunas variables que pueden ayudar a trabajar mejor estos contenidos. En esta asignatura se ha tenido la oportunidad de trabajar de forma paralela con dos grupos de alumnos/as a los que se les ha facilitado una batería de recursos online diferentes. A través de una encuesta a ambos grupos se ha recogido el uso y la valoración que hacían de los recursos multimedia disponibles para el apoyo al aprendizaje de las herramientas informáticas necesarias para el desarrollo de los ejercicios y tareas asignadas en la asignatura.

Palabras clave: recursos didácticos, aprendizaje autónomo, evaluación, rúbricas, TICs, recursos online. 


\section{Introducción}

Ya es una realidad que para la adecuación de la enseñanza al Espacio Europeo de Educación Superior (EEES) toda la actividad docente se debe dirigir a conseguir que el alumno tenga un papel mucho más activo en su proceso de aprendizaje. Dentro de esta meta y como reto pedagógico es fundamental implicar al estudiante en este cambio. Y como indica Claux (2001), es en este momento donde la labor del profesor debe facilitar dicha transformación, convirtiéndose en un guía, o un apoyo, durante la etapa formativa del alumno/a. Proceso donde el alumno/a debe de aprender a aprender, pero siempre tutelado por la figura del profesor (Díaz-Barriga, 2002; Van Petegem y Vanhoof, 2005).

A lo largo de estos años han proliferado numerosas tendencias en innovaciones metodológicas e investigaciones en educación que nos señalan la necesidad a cambiar las formas de enseñanza y evaluación utilizadas tradicionalmente (Arribas, Carabias y Monreal, 2010; Gessa 2011; Ureña y Ruiz, 2012; Fraile et al, 2013; Rodríguez et al, 2013; Hamodi et al, 2014; Nuñez et al, 2014; Hamodi et al, 2015; Hortigüela-Alcalá et al, 2015).

Así mismo y dentro de esta evidencia, el uso de materiales multimedia ya ha demostrado que favorece el proceso de aprendizaje (Marquès, 2000) y es parte de esas metodologías, donde la implicación activa del estudiante le permite alcanzar mayor efectividad en su formación (McKeachie, 1986). Pero todas ellas ya no pueden considerarse como nuevas. Y en ese sentido, la enseñanza universitaria debe buscar, de forma constante, estrategias didácticas que resulten atractivas al alumno/a a través no solo de la creación de nuevos materiales de apoyo, sino con la mejora constante de los que ya existen. Proceso de adaptación en el que nos debemos preguntar constantemente si estas estrategias son las más adecuadas y si sus contendidos se adaptan correctamente a los estudiantes a las que van encaminadas. No olvidando que debemos de considerar la diferencia que podemos tener entre los alumnos/as que conforman el grupo, donde se incluyen los considerados como nativos digitales que son aquellos nacidos en la era de las TIC y los que han nacido anteriormente como son los inmigrantes digitales y a los que se les exige su adaptación (Prensky, 2001). Todos ellos conforman las generaciones denominadas como Baby Boomers, la Generación X, Y o Z-Generación y Generación Net (Dávila, 2006).

Esta última premisa nos rebela un posible problema y es la diferencia de nivel que el alumnado puede tener del conocimiento y uso de las herramientas informáticas que se emplean en la asignatura de Fotografía y Documentación Aplicada al Estudio de los Bienes Culturales de tercero de Grado en Conservación y Restauración de Bienes Culturales, Facultad de Bellas Artes, Universitat Politècnica de València. Asignatura que tiene una carga lectiva muy significativa en el uso de herramientas informáticas para su desarrollo. Esta diferencia de nivel implica que algunos alumnos tengan suficiente con las explicaciones que los profesores dan en el aula sobre la herramienta, mientras que otros alumnos requieran de materiales complementarios y trabajo en casa para poder aprender correctamente las herramientas y ejecutar sus tareas.

Para solucionar este problema, los profesores prepararon una serie de materiales de apoyo con acceso a través de las herramientas de PoliformaT (descritos en sección 3.1). Para no solo conocer sino también comprobar si este material de apoyo era útil para resolver este problema se hicieron dos grupos de alumnos/as con diferentes planteamientos docentes; por un lado, un grupo que solo contaba con material de apoyo tipo documentos pdf, a diferencia de otro grupo que contaba además con una amplia batería de recursos complementarios online.

A través del sistema de evaluación y valoración que se propone, buscamos la capacidad de empatía que nos puede ayudar a mejorar el material usado como refuerzo (Zowghi y Paryani, 2003). Valoraciones que 
también deben contar y conocer el grado de satisfacción que tienen los alumnos con los recursos didácticos ofrecidos en una asignatura (Moltó, 2012).

Para ello, la valoración de los materiales se hará a través de una encuesta regida por la escala Likert. Dado que este método de medición es uno de los más utilizados por los investigadores con el objetivo de evaluar la opinión y actitudes de las personas. Destacando que una de las metas que se persigue es presentar una metodología que no solo sea sencilla sino rápida en su aplicación. La valoración pretendemos que no solo nos ayude a mejorar los materiales empleados, sino que nos aleje de otro de los posibles problemas como es la desmotivación y aburrimiento por parte de los alumnos (Brown, Collins \& Duguid, 1989).

El resto del artículo está estructurado a través de varios puntos. La Sección 2 presenta los diferentes hitos y metas que persigue nuestro trabajo. La Sección 3 destalla tanto el ámbito de aplicación como los elementos empleados para la valoración, más los aspectos técnicos de los mismos. En la Sección 4 se analizan los resultados obtenidos a través de distintas perspectivas y la Sección 5 recoge las conclusiones alcanzadas y plantea los próximos retos para abordar en futuros trabajos.

\section{Objetivos}

Ambas propuestas, evaluación y valoración de los recursos que pusimos en marcha, intentaban trabajar con un término que Marton y Säljö (1976a, 1976b) desarrollaron en sendos estudios como es enfoque del aprendizaje. Este término se define como los procesos de aprendizaje que emergen de la propia percepción que el estudiante tiene de las tareas que ha realizado en la asignatura. Valoraciones que van a tener presentes una gran multitud de variables, como; la naturaleza de la tarea, el sistema de evaluación, el método de enseñanza, la relevancia del curso, el interés del estudiante o su estilo de aprendizaje, todas desarrolladas por la percepción del estudiante (Laurillard, 1979; Hernández et al. 2002).

Por lo que los objetivos marcados han sido:

1. Evaluar tanto la utilidad en el aprendizaje como el uso realizado de recursos multimedia aportados.

2. Analizar el grado de satisfacción de los alumnos/as con los recursos utilizados/disponibles y sus propuestas de mejora.

3. Conocer el grado de satisfacción con los sistemas de evaluación.

4. Comparar resultados para definir el planteamiento de nuevas actuaciones relacionadas con la preparación de nuevos materiales orientados a la mejora del aprendizaje.

5. Conocer la percepción que los alumnos tienen de la exigencia (mas o menos dificultad y mucho o poco tiempo empleado) que les supone la realización de las tareas de la asignatura.

\section{Desarrollo de la innovación}

El estudio se realizó en la asignatura Fotografía y Documentación Aplicada al Estudio de los Bienes Culturales de tercero de Grado en Conservación y Restauración de Bienes Culturales, Facultad de Bellas Artes, Universitat Politècnica de València).

Asignatura cuatrimestral (primer cuatrimestre) de carácter obligatorio, cuenta con 9 créditos prácticos. El objetivo principal es instruir al alumno en el uso de la fotografía y los recursos informáticos entendidos como métodos científicos de análisis no invasivo. A través de diversas herramientas se intenta iniciar al estudiante en el ámbito de la documentación de obras de arte con fines conservativos o restaurativos. La 
asignatura se divide en 3 bloques de 3 créditos bien diferenciados: teoría, práctica de fotografía (en el aula de fotografía) y práctica informática (en el aula informática).

De forma coordinada, el alumno/a va desarrollando una serie de actividades que comienzan con los ejercicios fotográficos planteados sobre obra o pieza que es caso de estudio (pintura de caballete, pintura mural, papel, u obra volumétrica como pueden ser elementos escultóricos u objetos arqueológicos), para continuar con la elaboración de sus diagramas de deterioro. Todo este material conforma informes técnicos de conservación y restauración. Siendo estas dos últimas partes donde hemos querido aplicar el análisis de valoración de los recursos empleados. Este estudio se centra en el uso y valoración del material empleado en las prácticas informáticas (clases semanales de $2 \mathrm{~h}$ ). Material que se distribuye en dos unidades didácticas (UD): UD3. Vectorización digital específica para la elaboración de mapas de daños y UD4. Maquetación de documentos de registro de intervenciones.

Debido al elevado numero de alumnos matriculados (70) y el número de ordenadores existentes en el aula informática, los alumnos/as se dividen en 3 grupos: grupo A (35 alumnos/as), grupo B (19 alumnos/as) y grupo C (16 alumnos/as). En función del número de alumnos, hemos establecido dos grupos; un grupo de control (GC) formado por el grupo A (35 alumnos/as) y un grupo de intervención (GI) que lo integran el grupo $\mathrm{B}$ y el grupo $\mathrm{C}$ (35 alumnos/as). Al GI se le proporcionó una serie de materiales complementarios/extras de apoyo online (descritos en sección 3.1) mientras que al grupo control no.

\subsection{Descripción de los materiales}

Para elaborar esta experiencia el GC contaba solo con el material que se proporcionaba en la misma clase, teniendo como base una batería de ejemplos de años anteriores y las lecciones teórico-prácticas realizadas durante las sesiones de clase. Además, este grupo no contó con una rúbrica, que sirviera de guía en el proceso de evaluación. En este caso, lo criterios de evaluación, que eran los mismos en ambos grupos, fueron expuestos por el profesor en una sesión ordinaria de clase. Esto contrasta con los recursos con los que contaba el grupo de intervención.

El GI, por su parte, contó además de los materiales del apartado de recursos, con un amplio abanico de materiales didácticos en la plataforma PoliformaT:

- Resúmenes de las Unidades Didácticas en Lessons que permiten organizar los apuntes de la asignatura de forma accesible y cómoda.

- Tests de evaluación/repaso mediante Kahoot, un servicio web de educación social y gamificada que se basa en la premisa de 'aprender divirtiéndose'. Una vez creado un test, los alumnos se unen a la actividad introduciendo un código PIN en la aplicación para móvil. De este modo, el móvil se convierte en un control remoto con el cual pueden responder a las preguntas fácilmente, mientras que en la pantalla se muestra la pregunta y la clasificación de los jugadores en función de las respuestas acertadas. Es importante matizar que Kahoot es una herramienta de refuerzo, pues las preguntas son demasiado cortas como para profundizar.

- Vídeo tutoriales para facilitar el aprendizaje autónomo de las herramientas a través de Videoapuntes en la plataforma UPV [Media]. Cabe destacar que este servicio ofrece la posibilidad de grabar las clases presenciales, así como grabar las sesiones de forma anticipada para ser usadas como material de aprendizaje autónomo, por lo que resulta una herramienta interesante para reforzar la asimilación de las herramientas.

Y como hemos adelantado, el GI ha contado con la rúbrica (Blanco, 2008) de evaluación a modo de herramienta de autocorrección dentro de la parte destinada a la maquetación de sus portafolios, cuyo uso valoraremos posteriormente en el análisis de los instrumentos (tabal 1). En esta rúbrica el alumno/a tiene la valoración de aspectos como; organización de los elementos en el diseño o maquetación, uso de los 
elementos de tipografía y encaje de las cajas de texto, inclusión de los elementos propios de una página, orden de los distintos elementos visuales, o una valoración sobre el su aspecto estético y contraste. Todas estas categorías están organizadas en 4 niveles con distinta valoración.

Tabla 1. Descripción de las preguntas del formulario para el grupo intervención (GI)

\begin{tabular}{|c|c|c|c|c|}
\hline Aspectos valorados & \multicolumn{4}{|c|}{ Puntuación total obtenida } \\
\hline \multirow{2}{*}{ Valoración global } & 50 puntos & 40 puntos & 30puntos & 20 puntos \\
\hline & MUY BUENA & BUENA & ACCEPTABLE & MEJORABLE \\
\hline $\begin{array}{c}\text { 1. Organización } \\
\text { adecuada de los } \\
\text { elementos (imágenes, } \\
\text { textos, pies de figura, } \\
\text { notas al pie, número } \\
\text { de página...) }\end{array}$ & $\begin{array}{l}\text { Incluye todos los } \\
\text { elementos de manera } \\
\text { muy bien organizada, } \\
\text { clara y coherente } \\
\mathbf{1 0} \text { puntos }\end{array}$ & $\begin{array}{l}\text { Incluye todos los } \\
\text { elementos de forma } \\
\text { organizada y clara } \\
\mathbf{8} \text { puntos }\end{array}$ & $\begin{array}{c}\text { Incluye todos los } \\
\text { elementos de manera } \\
\text { poco organizada y clara } \\
\mathbf{6} \text { puntos }\end{array}$ & $\begin{array}{l}\text { No incluye todos los } \\
\text { elementos de forma } \\
\text { organizada y clara } \\
4 \text { puntos }\end{array}$ \\
\hline $\begin{array}{c}\text { 2. Tipologías e } \\
\text { interlineados } \\
\text { adecuados y reglas } \\
\text { ortográficas correctas }\end{array}$ & $\begin{array}{c}\text { Las tipologías e } \\
\text { interlineados elegidos } \\
\text { son muy adecuados y } \\
\text { diferentes para cada } \\
\text { categoría. Las reglas } \\
\text { ortográficas son } \\
\text { correctas } \\
\mathbf{1 0} \text { puntos }\end{array}$ & $\begin{array}{c}\text { Las tipologías e } \\
\text { interlineados elegidos } \\
\text { son adecuados y } \\
\text { diferentes para alguna } \\
\text { categoría, pero no } \\
\text { todas. Las reglas } \\
\text { ortográficas son } \\
\text { correctas } \\
\mathbf{8} \text { puntos }\end{array}$ & $\begin{array}{c}\text { Las tipologías e } \\
\text { interlineados elegidos } \\
\text { son adecuados, pero no } \\
\text { diferentes entre } \\
\text { categorías. Hay } \\
\text { algunos errores } \\
\text { ortográficos y } \\
\text { gramaticales. } \\
\mathbf{6} \text { puntos }\end{array}$ & $\begin{array}{c}\text { Las tipologías e } \\
\text { interlineados elegidos } \\
\text { no son adecuados ni } \\
\text { diferentes entre } \\
\text { categorías. Hay } \\
\text { muchos errores } \\
\text { ortográficos y } \\
\text { gramaticales. } \\
\mathbf{4} \text { puntos }\end{array}$ \\
\hline $\begin{array}{l}\text { 3. Inclusión de todos } \\
\text { los elementos } \\
\text { necesarios: portada, } \\
\text { índice, introducción, } \\
\text { número de página, } \\
\text { imágenes, anexos... }\end{array}$ & $\begin{array}{c}\text { Incluye todos los } \\
\text { elementos necesarios } \\
\text { de manera muy bien } \\
\text { organizada, clara y } \\
\text { coherente } \\
\mathbf{1 0} \text { puntos } \\
\end{array}$ & $\begin{array}{c}\text { Incluye todos los } \\
\text { elementos necesarios } \\
\text { de forma organizada y } \\
\text { clara } \\
\mathbf{8} \text { puntos }\end{array}$ & $\begin{array}{l}\text { Incluye todos los } \\
\text { elementos necesarios, } \\
\text { pero de manera poco } \\
\text { organizada y clara } \\
\mathbf{6} \text { puntos }\end{array}$ & $\begin{array}{c}\text { No incluye todos los } \\
\text { elementos necesarios, y } \\
\text { los que incluyen están } \\
\text { mal organizados y poco } \\
\text { claros } \\
\mathbf{4} \text { puntos } \\
\end{array}$ \\
\hline $\begin{array}{l}\text { 4. Orden, categorías y } \\
\text { jerarquías claras y } \\
\text { visuales }\end{array}$ & $\begin{array}{l}\text { El orden de las } \\
\text { categorías está bien } \\
\text { jerarquizado y } \\
\text { estructurado de forma } \\
\text { lógica, visual y } \\
\text { creativa } \\
\mathbf{1 0} \text { puntos }\end{array}$ & $\begin{array}{c}\text { El orden de las } \\
\text { categorías está } \\
\text { jerarquizado y } \\
\text { estructurado de forma } \\
\text { lógica y visual } \\
\mathbf{8} \text { puntos }\end{array}$ & $\begin{array}{c}\text { El orden de las } \\
\text { categorías está } \\
\text { jerarquizado y } \\
\text { estructurado, pero no se } \\
\text { entiende claramente y } \\
\text { no es visual } \\
\mathbf{6} \text { puntos }\end{array}$ & $\begin{array}{c}\text { El documento esta } \\
\text { desordenado, las } \\
\text { categorías son } \\
\text { confusas, poco visuales } \\
\text { y nada creativas, no } \\
\text { entendiéndose bien su } \\
\text { jerarquía. Falta una } \\
\text { buena estructuración. } \\
\mathbf{4} \text { puntos }\end{array}$ \\
\hline 5. Estética y contraste & $\begin{array}{l}\text { La estética y contraste } \\
\text { incluye elementos de } \\
\text { color y figuras } \\
\text { geométricas creativas } \\
\text { que ayudan a la lectura } \\
\text { y comprensión } \\
\text { consiguiendo interesar } \\
\text { al lector y atraer su } \\
\text { atención } \\
\mathbf{1 0} \text { puntos }\end{array}$ & $\begin{array}{l}\text { La estética y contraste } \\
\text { es correcta. Incluye } \\
\text { elementos de color o } \\
\text { figuras geométricas que } \\
\text { mejoran la lectura } \\
\mathbf{8} \text { puntos }\end{array}$ & $\begin{array}{l}\text { La estética y contraste } \\
\text { es mejorable, aunque } \\
\text { incluye algún elemento } \\
\text { de color } \\
\mathbf{6} \text { puntos }\end{array}$ & $\begin{array}{l}\text { No hay elementos de } \\
\text { color, figuras } \\
\text { geométricas, contraste, } \\
\text { etc. que ayuden a la } \\
\text { lectura y comprensión. } \\
\text { La estética es aburrida } \\
\text { y monótona } \\
\mathbf{4} \text { puntos }\end{array}$ \\
\hline
\end{tabular}

\subsection{Instrumentos de valoración}

Para poder dar respuesta a nuestros objetivos y conocer el grado de satisfacción de los alumnos con los recursos utilizados se ha elaborado un cuestionario a través de Google Forms. Los formularios no solo han perseguido ofrecer una información cuantitativa a las preguntas efectuadas, sino que también han perseguido una valoración cualitativa de las mismas (Starks y Trinidad, 2007). Este segundo aspecto, como apunta Klopper (2008), aporta datos y participa en la generación de hipótesis para posteriores trabajos en diferentes contextos como la docencia o la investigación, entre otros. La importancia de la 
metodología cualitativa radica en que aporta datos para ayudar a comprender el qué, el cómo y por qué (Sheldon, 2005).

Se prepararon dos formularios ligeramente diferentes, uno para cada uno de los grupos, GC y GI. El formulario del GC (tabla 2), estaba formado por 8 preguntas, 7 de respuesta dirigida (1-5) y una pregunta final de respuesta abierta. La primera pregunta está enfocada a evaluar la utilidad de los recursos didácticos disponibles (primer objetivo propuesto). Las dos primeras preguntas (1 y 2), planteaban un ámbito más amplio y general en la consulta, a diferencia de las tres siguientes $(3,4$ y 5$)$ cuyo objetivo era conocer la percepción que los alumnos tienen de la exigencia de las tareas planteadas. Analizado diferentes variables como; mayor o menor dificultad y mayor o menor tiempo empleado en el desarrollo de las tareas. La cuestión relacionada con dinámica de entrega y evaluación de los ejercicios/tareas planteadas en la asignatura relacionado también fue evaluada con dos preguntas (6 y 7). El cuestionario se completaba con una pregunta de respuesta abierta en la que se pretendía conocer los aspectos que se podrían mejorar.

Tabla 2. Descripción de las preguntas del formulario para el grupo control (GC)

\begin{tabular}{|l|l|l|}
\hline $\mathbf{N}^{\mathbf{0}}$ & Enunciado preguntas grupo control (GC) & Objetivo \\
\hline 1 & ¿El material que se dispone en los recursos de la asignatura es suficiente? & 1 \\
\hline 2 & $\begin{array}{l}\text { Los recursos multimedia como Polimedia, Lessons, Kahoot, ¿consideras que } \\
\text { serían de ayuda para la asignatura? }\end{array}$ & 2 \\
\hline 3 & $\begin{array}{l}\text { Las herramientas (Corel e InDesign) empleadas para acometer los ejercicios } \\
\text { planteados en la asignatura son fáciles de manejar }\end{array}$ & 5 \\
\hline 4 & $\begin{array}{l}\text { ¿Consideras que la cantidad de tiempo que has invertido para realizar la tarea ha } \\
\text { sido adecuada? }\end{array}$ & 5 \\
\hline 5 & $\begin{array}{l}\text { Respecto a la percepción sobre la complejidad o dificultad en la realización de } \\
\text { las tareas, ¿consideras que han sido difíciles o complicadas? }\end{array}$ & 5 \\
\hline 6 & $\begin{array}{l}\text { ¿Consideras que la dinámica de corrección y reentrega de las tareas ha sido útil } \\
\text { en tu aprendizaje? }\end{array}$ & 3 \\
\hline 7 & $\begin{array}{l}\text { ¿Consideras que sería de ayuda disponer de la rúbrica (tabla con valores para } \\
\text { corregir los ejercicios de la asignatura) para tu aprendizaje? }\end{array}$ & 3 \\
\hline 8 & $\begin{array}{l}\text { ¿Qué aspectos crees que se pueden mejorar en la asignatura? Escribe tus } \\
\text { propuestas o sugerencias. }\end{array}$ & 2 \\
\hline
\end{tabular}

Con el objetivo de comparar y analizar las respuestas, para el GI el cuestionario contaba con una serie de preguntas que presentaban varios enunciados comunes al GC (7 preguntas comunes) y una serie de enunciados específicos (preguntas 2 y 7 del GC y 2, 8, 9 del GI) para valorar el material adicional del que este grupo disponía (tabla 3). Las preguntas con enunciados comunes permitían volver a valorar la percepción general del alumno/a, el nivel de exigencia y también se les abría la posibilidad a hacer sugerencias. En relación con las preguntas específicas para el GI el interés se centraba en que señalaran de los recursos empleados el que más grado de satisfacción presentaba (3) y, por otro lado, conocer el grado de satisfacción sobre la rúbrica preparada para la evaluación de los ejercicios (8 y 9). 
Tabla 3. Descripción de las preguntas del formulario para el grupo intervención (GI)

\begin{tabular}{|c|l|c|}
\hline $\mathbf{N}^{\mathbf{0}}$ & \multicolumn{1}{|c|}{ Enunciado preguntas grupo intervención (GI) } & Objetivo \\
\hline 1 & ¿El material que se dispone en los recursos de la asignatura es suficiente? & 1 \\
\hline 2 & $\begin{array}{l}\text { ¿Crees que los recursos multimedia como Polimedia, Lessons, Kahoot han sido } \\
\text { útiles en tu aprendizaje? }\end{array}$ & 2 \\
\hline 3 & $\begin{array}{l}\text { De los siguientes 4 recursos multimedia de la asignatura indica cuál te ha sido } \\
\text { más útil: a) Polimedia (vídeos), b) Lessons (PoliformaT), c) Kahoot y d) Carpeta } \\
\text { de recursos (PoliformaT) }\end{array}$ & 5 \\
\hline 4 & $\begin{array}{l}\text { ¿Las herramientas (Corel e InDesign) empleadas para acometer los ejercicios } \\
\text { planteados en la asignatura consideras que son fáciles de manejar? }\end{array}$ & 5 \\
\hline 5 & $\begin{array}{l}\text { ¿Consideras que la cantidad de tiempo que has invertido para realizar las tareas ha } \\
\text { sido adecuada? }\end{array}$ & $\begin{array}{l}\text { Respecto a la percepción sobre la complejidad o dificultad en la realización de las } \\
\text { tareas, ¿consideras que han sido difíciles o complicadas? }\end{array}$ \\
\hline 7 & $\begin{array}{l}\text { ¿Consideras que la dinámica de corrección y reentrega de las tareas ha sido útil en } \\
\text { tu aprendizaje? }\end{array}$ & 3 \\
\hline 8 & ¿Disponer de la rúbrica ha ayudado en tu aprendizaje? & 3 \\
\hline 9 & ¿Los valores e información disponible en las rúbricas son suficientes? & 3 \\
\hline 10 & $\begin{array}{l}\text { ¿Qué aspectos crees que se pueden mejorar en la asignatura? Escribe tus } \\
\text { propuestas o sugerencias. }\end{array}$ & 2 \\
\hline
\end{tabular}

Tal y como hemos apuntado anteriormente, para las preguntas se ha empleado la escala Likert que se distingue de otro tipo de cuestionarios porque mide el grado en que el encuestado está de acuerdo o en desacuerdo con cada consulta. Aunque se pueden generar modelos que contemplan de 4 a 7 posibilidades de respuesta, se empleó el modelo de cinco variantes de respuestas, que es la opción más habitual. Concretamente consiste en estas opciones: 1. Totalmente en desacuerdo; 2. En desacuerdo; 3. Ni de acuerdo ni en desacuerdo; 4. De acuerdo; 5 . Totalmente de acuerdo.

Estos formularios con carácter voluntario y anónimo se pasaron en diciembre (curso 2019/2020) para que los alumnos tuvieran tiempo de conocer y consultar los distintos recursos facilitados.

\section{Resultados}

La encuesta fue contestada por $28(80,0 \%)$ en el caso del GC (véase Tabla 4$)$ y por $30(86,0 \%)$ (véase Tabla 5) en el caso del GI. Tras el análisis de los datos ofrecidos por ambas encuestas, nos llama la atención la poca diferencia que existen entre los datos de ambas en las preguntas comunes. En relación con la primera pregunta, sobre sí el material con que disponen es suficiente y solo atendiendo al nivel más alto de valoración, el 39,6\% del GC se muestra muy de acuerdo con la afirmación, mientras que del GI parece más exigente en este tema pues el porcentaje es del $30 \%$ es el que está de acuerdo. Incluso con la suma de los valores 4 y 5 de la escala no existen diferencias significativas (fig.1).

Respecto a su utilidad didáctica (“¿Crees que los recursos multimedia (Polimedia, Lessons, Kahoot) han sido útiles en tu aprendizaje?") pregunta 2 del GI, un 53,3\% del grupo valora positivamente haber contado con este apoyo para su aprendizaje, que junto con el $30,0 \%$ que se muestra de acuerdo, conforman un $83,3 \%$ de todo el grupo (fig.2).

En el GC se les formuló una cuestión similar, indicándoles si consideraban que este tipo de recursos podría ayudar. En este caso, un importante número de los encuestados en el GC no tenían una opinión formada $(42,9 \%)$, pero el resto se decantaba hacia la afirmación de que sí podrían ser de ayuda $(53,5 \%)$. 
Los recursos multimedia que empleo, ¿son los más adecuados?

Tabla 4. Resumen de los resultados completos de la encuesta realizada al Grupo Intervención

\begin{tabular}{|c|c|c|c|c|c|}
\hline Grupo intervención & I & 2 & 3 & 4 & 5 \\
\hline $\begin{array}{l}\text { I. ¿El material que se dispone en los recursos de la } \\
\text { asignatura es suficiente? }\end{array}$ & $3(10 \%)$ & $0(0 \%)$ & $4(13,3 \%)$ & $14(46,7 \%)$ & $9(30 \%)$ \\
\hline $\begin{array}{l}\text { 2. Los recursos multimedia como Polimedia, Lessons, } \\
\text { Kahoot, ¿consideras que serían de ayuda para la asignatura? }\end{array}$ & I (3,3\%) & $0(0 \%)$ & $4(13,3 \%)$ & $9(30 \%)$ & $16(53,3 \%)$ \\
\hline $\begin{array}{l}\text { 3. De los siguientes } 4 \text { recursos multimedia de la asignatura } \\
\text { indica cuál te ha sido más útil: }\end{array}$ & \multicolumn{5}{|c|}{$\begin{array}{c}\text { Kahoot (10\%) / Polimedia vídeos (13,3\%) } \\
\text { / Carpeta de recursos PoliformaT (26,7\%) / Lessons PoliformaT (50\%) }\end{array}$} \\
\hline $\begin{array}{l}\text { 4. Las herramientas (Corel e InDesign) empleadas para acometer } \\
\text { los ejercicios planteados en la asignatura son fáciles de manejar }\end{array}$ & $0(0 \%)$ & $0(0 \%)$ & II (36,7\%) & $17(56,7 \%)$ & $2(6,7 \%)$ \\
\hline $\begin{array}{l}\text { 5. ¿Consideras que la cantidad de tiempo que has invertido para } \\
\text { realizar la tarea ha sido adecuado? }\end{array}$ & $0(0 \%)$ & $2(6,7 \%)$ & $4(13,3 \%)$ & $13(43,3 \%)$ & II $(36,7 \%)$ \\
\hline $\begin{array}{l}\text { 6. Respecto a la percepción sobre la complejidad o dificultad en } \\
\text { la realización de las tareas, i consideras que han sido difíciles o } \\
\text { complicadas? }\end{array}$ & $3(10 \%)$ & $7(23,3 \%)$ & II (36,7\%) & $9(30 \%)$ & $0(0 \%)$ \\
\hline $\begin{array}{l}\text { 7. ¿Consideras que la dinámica de corrección y reentrega de las } \\
\text { tareas ha sido útil en tu aprendizaje? }\end{array}$ & $0(0 \%)$ & $0(0 \%)$ & $3(10 \%)$ & $9(30 \%)$ & $18(60 \%)$ \\
\hline 8. ¿Disponer de la rúbrica ha ayudado en tu aprendizaje? & $0(0 \%)$ & I (3,3\%) & $4(13,3 \%)$ & $9(30 \%)$ & $16(53,3 \%)$ \\
\hline $\begin{array}{l}\text { 9. ¿Los valores e información disponible en las rúbricas es } \\
\text { suficiente? }\end{array}$ & $0(0 \%)$ & $2(6,7 \%)$ & $8(26,7 \%)$ & II (36,7 \%) & $9(30 \%)$ \\
\hline
\end{tabular}

L.Totalmente en desacuerdo; 2 . En desacuerdo; 3 . Ni de acuerdo ni en desacuerdo;

4. De acuerdo; 5. Totalmente de acuerdo.

Tabla 5. Resumen de los resultados completos de la encuesta realizada al Grupo Control

\begin{tabular}{|c|c|c|c|c|c|}
\hline Grupo control & 1 & 2 & 3 & 4 & 5 \\
\hline $\begin{array}{l}\text { I. ¿El material que se dispone en los recursos de la } \\
\text { asignatura es suficiente? }\end{array}$ & $0(0 \%)$ & I (3,6\%) & $8(28,6 \%)$ & $8(28,6 \%)$ & II (39,3\%) \\
\hline $\begin{array}{l}\text { 2. Los recursos multimedia como Polimedia, Lessons, } \\
\text { Kahoot, ¿consideras que serían de ayuda para la asignatura? }\end{array}$ & $0(0 \%)$ & I (3,6\%) & $12(42,9 \%)$ & $9(32,1 \%)$ & $6(21,4 \%)$ \\
\hline $\begin{array}{l}\text { 3. Las herramientas (Corel e InDesign) empleadas para acometer } \\
\text { los ejercicios planteados en la asignatura son fáciles de manejar }\end{array}$ & $0(0 \%)$ & $3(10,7 \%)$ & $4(14,3 \%)$ & $16(57,1 \%)$ & $5(17,9 \%)$ \\
\hline $\begin{array}{l}\text { 4. ¿Consideras que la cantidad de tiempo que has invertido para } \\
\text { realizar la tarea ha sido adecuado? }\end{array}$ & $0(0 \%)$ & I (3,6\%) & $8(28,6 \%)$ & II $(39,3 \%)$ & $8(28,6 \%)$ \\
\hline $\begin{array}{l}\text { 5. Respecto a la percepción sobre la complejidad o dificultad en } \\
\text { la realización de las tareas, i consideras que han sido difíciles o } \\
\text { complicadas? }\end{array}$ & $0(0 \%)$ & $6(21,4 \%)$ & $12(42,9 \%)$ & $9(32,1 \%)$ & I (3,6\%) \\
\hline $\begin{array}{l}\text { 6. ¿Consideras que la dinámica de corrección y reentrega de las } \\
\text { tareas ha sido útil en tu aprendizaje? }\end{array}$ & $0(0 \%)$ & $0(0 \%)$ & $0(0 \%)$ & $10(35,7 \%)$ & $18(64,3 \%)$ \\
\hline $\begin{array}{l}\text { 7. ¿Qué aspectos crees que se pueden mejorar en la asignatura? } \\
\text { Escribe tus propuestas o sugerencias. }\end{array}$ & $0(0 \%)$ & I (3,6\%) & $7(25 \%)$ & II (39,3\%) & $9(32,1 \%)$ \\
\hline
\end{tabular}

I.Totalmente en desacuerdo; 2 . En desacuerdo; 3 . Ni de acuerdo ni en desacuerdo;

4. De acuerdo; 5. Totalmente de acuerdo. 


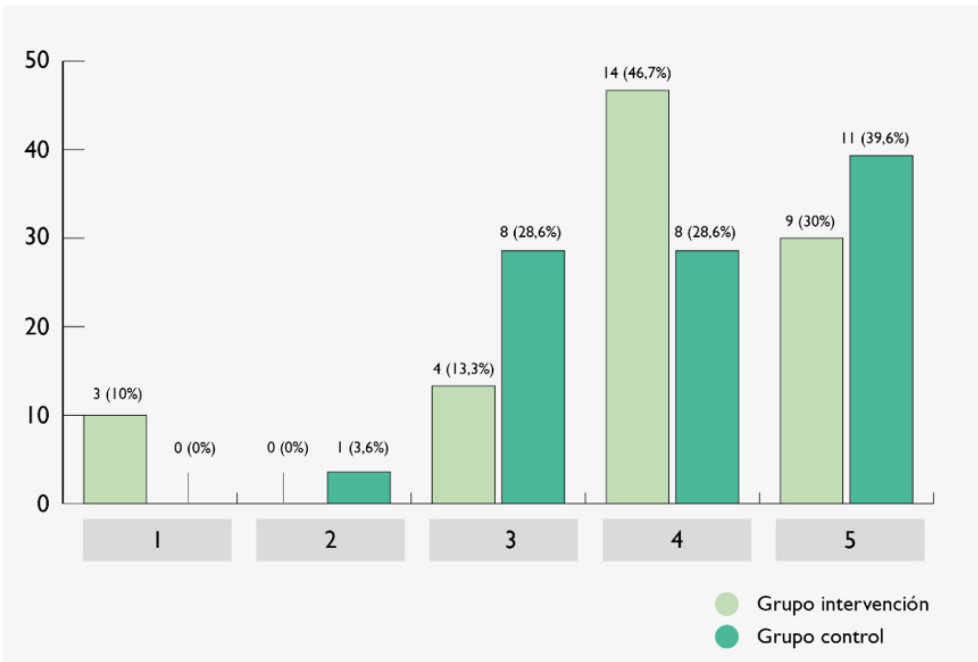

Fig. 1 Gráfica con la respuesta a la pregunta 1 “El material que se dispone en los recursos de la asignatura es suficiente? (Grupo control y grupo intervención)

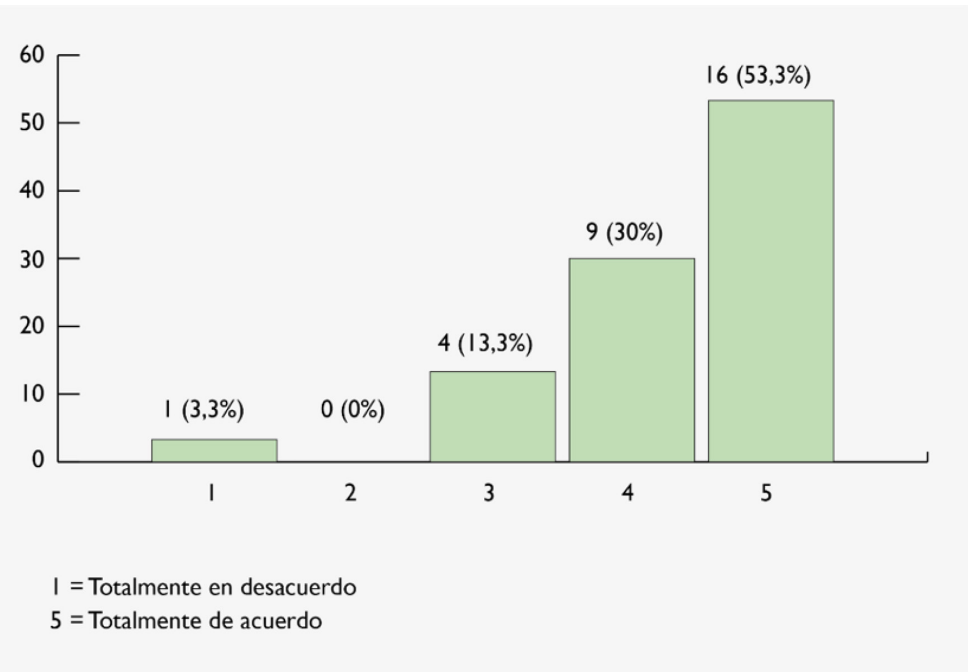

Fig. 2 ¿Crees que los recursos multimedia (Polimedia, Lessons, Kahoot) han sido útiles en tu aprendizaje? (Pregunta 2, Grupo intervención)

Resulta llamativo el resultado de la pregunta 3 del GI referente a "De los siguientes 4 recursos multimedia de la asignatura indica cuál te ha sido más útil: a) Polimedia (vídeos); b) Lessons (PoliformaT); c) Kahoot y d) Carpeta de recursos (PoliformaT)". En este caso, sería sencillo presuponer que este alumnado puede preferir herramientas asociadas a un mayor dinamismo e interacción en el aprendizaje, como los tests gamificados de Kahoot o los videotutoriales para aprender software en Polimedia. No obstante, un 50,0\% del alumnado prefiere los apuntes elaborados disponibles en Lessons frente al 26,7\% que elige el material de la carpeta recursos, el 13,3\% que escoge los vídeo tutoriales de Polimedia y tan solo el 10,0\% que opta por los tests gamificados de Kahoot (fig.3). 


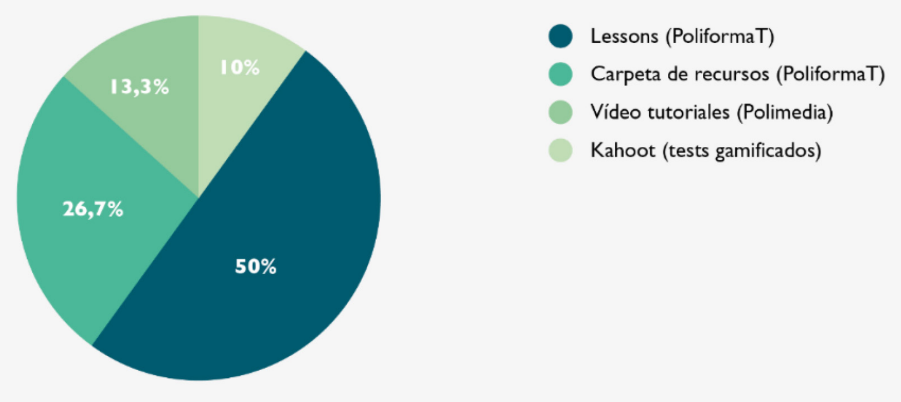

Fig. 3 Gráfica con la respuesta a la pregunta 3 del Grupo intervención: "De los siguientes 4 recursos multimedia de la asignatura indica cuál te ha sido más útil: a) Polimedia (vídeos); b) Lessons (PoliformaT); c) Kahoot y d) Carpeta de recursos (PoliformaT)”.

De forma paralela, hemos contado con los datos de acceso a los recursos, concretamente los datos obtenidos mediante la herramienta Estadísticas (PoliformaT). Estos datos pueden dar una referencia de escala del uso que se hace de los recursos complementarios (objetivo 1). Este recurso ha registrado un número de accesos total a Lessons (PoliformaT) de 2977 visitas, solo del GI, durante los meses que dura la asignatura (septiembre-enero) donde se observa como hay un crecimiento en el número de accesos importante desde septiembre (401), octubre (633) con un máximo de accesos en noviembre (1028) y un posterior descenso importante en diciembre (425) y en enero (490) posiblemente debido al periodo de evaluación. Por otro lado, se observa que hay una consulta muy variable de estos recursos por parte del alumnado (GI) encontrando alumnos que han entrado tan solo 2 veces a Lessons y alumnos que han consultado este recurso hasta 385 veces a lo largo del curso. Si comparamos las visitas que los estudiantes han realizado al PoliformaT de la asignatura observamos como el número medio de visitas por alumno es mucho mayor en el GI (81,23visitas) mientras que en el GC el numero medio de visitas es de 5,14.

En relación con la pregunta de la idoneidad de las herramientas informáticas planteadas para poder resolver los ejercicios (preguntas $3 \mathrm{GC}$ y $4 \mathrm{GI}$ ), ambos grupos han considerado que son las más adecuadas. Nuevamente la suma de los dos valores de mayor rango en la encuesta es similar y mayoritaria, con un 75,0 \% en el GC y un 63,4 \% en el GI (fig.4).

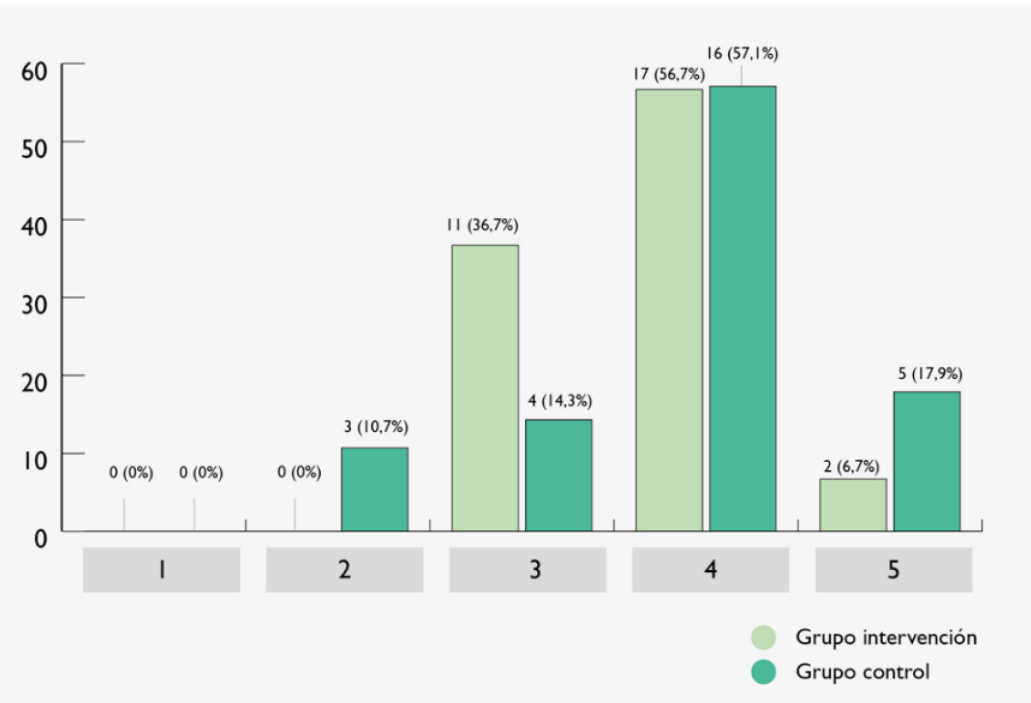

Fig. 4 Gráfica con la respuesta a la pregunta (preguntas 3 GC y 4 GI): "Las herramientas (Corel e InDesign) empleadas para acometer los ejercicios planteados en la asignatura son fáciles de manejar”. 
Aunque es difícil de valorar tampoco se ven diferencias significativas entre ambos grupos en relación con la consideración del tiempo empleado (fig.5) para resolver los ejercicios propuestos (preguntas 4 GC y 5 GI) o la percepción de la dificultad (fig.6) para ejecutar los ejercicios propuestos (preguntas 5 GC y 6 GI), e incluso la pregunta referida a la dinámica de corrección y retroalimentación propuesta (preguntas $6 \mathrm{GC}$ y 7 GI). En esta última pregunta, los porcentajes en el rango de más valor fueron de $66,3 \%$ en el GC y de $60,0 \%$ en el GI.

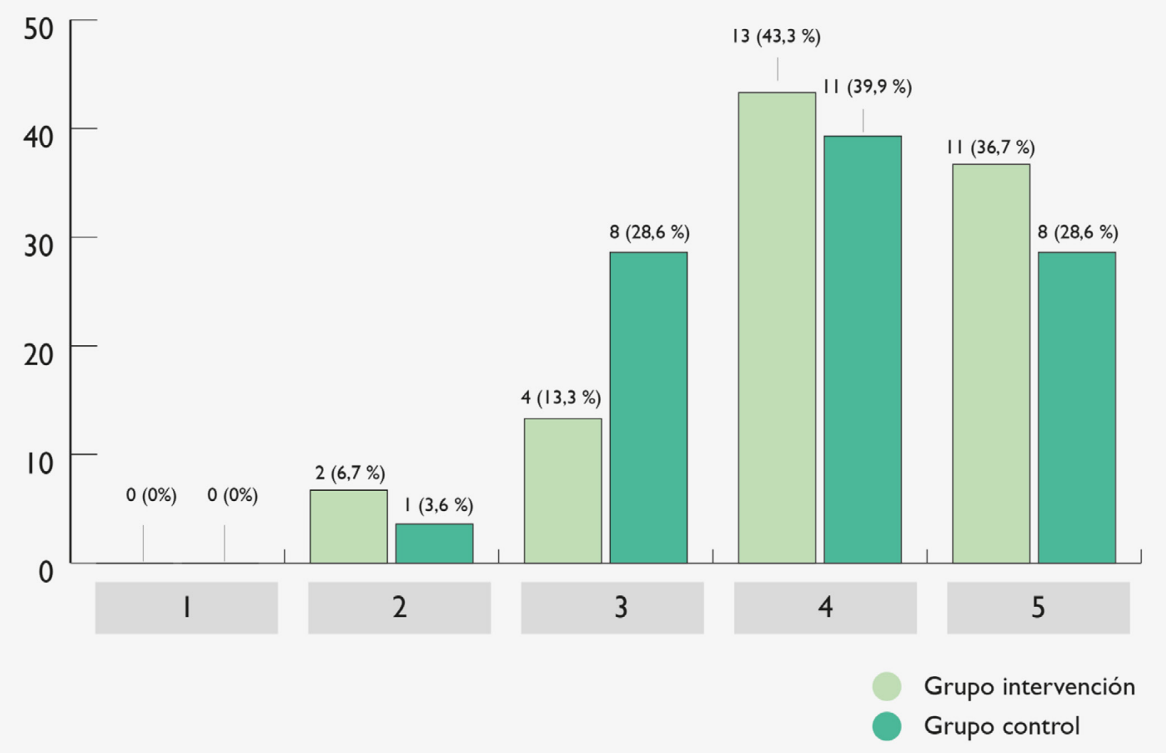

Fig. 5 Gráfica con la respuesta a la pregunta (preguntas 4 GC y 5 GI): “Consideras que la cantidad de tiempo que has invertido para realizar la tarea ha sido adecuada?".

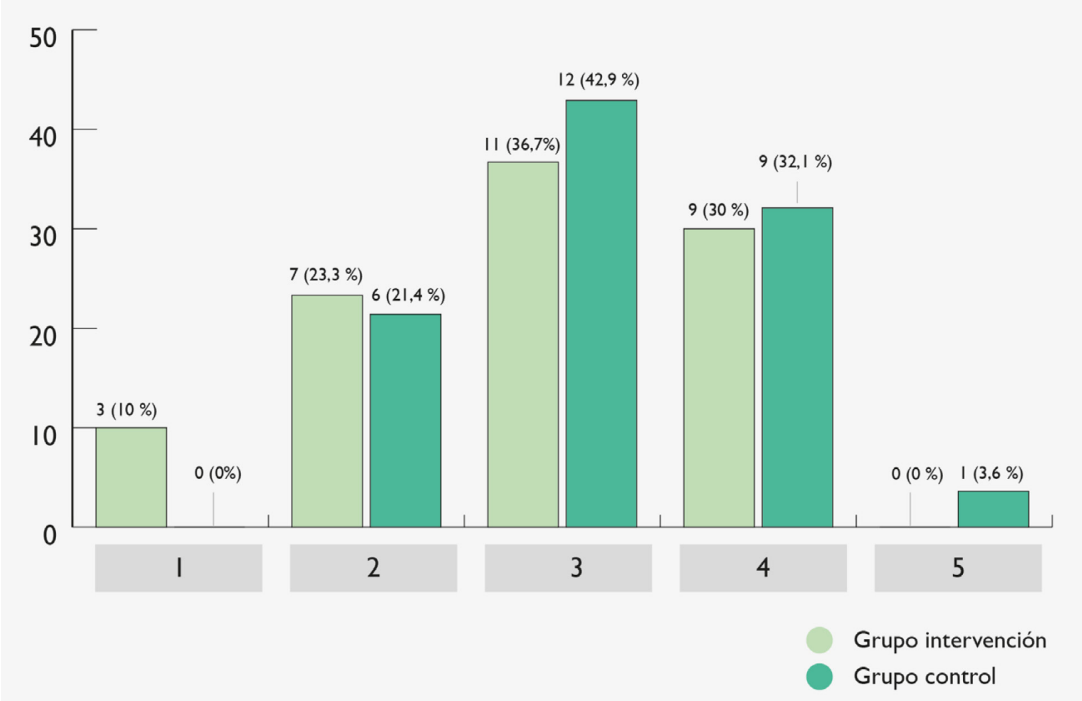

Fig. 6 Gráfica con la respuesta a la pregunta (preguntas 5 GC y 6 GI): "Respecto a la percepción sobre la complejidad o dificultad en la realización de las tareas, ¿consideras que han sido dificiles o complicadas?”. 
Sobre el tema la evaluación a través de la rúbrica, en el caso del GC (pregunta 7 GC) hay una tendencia clara hacia la afirmación de qué sí la hubieran necesitado, pues la suma de los valores de los dos rangos más altos se alcanza un 71,4\% siendo significativo, el $25 \%$ de los encuestados que no tienen una opinión formada sobre el tema (fig. 7). Lo que sí es cierto es que no es significativa la respuesta negativa en este tema.

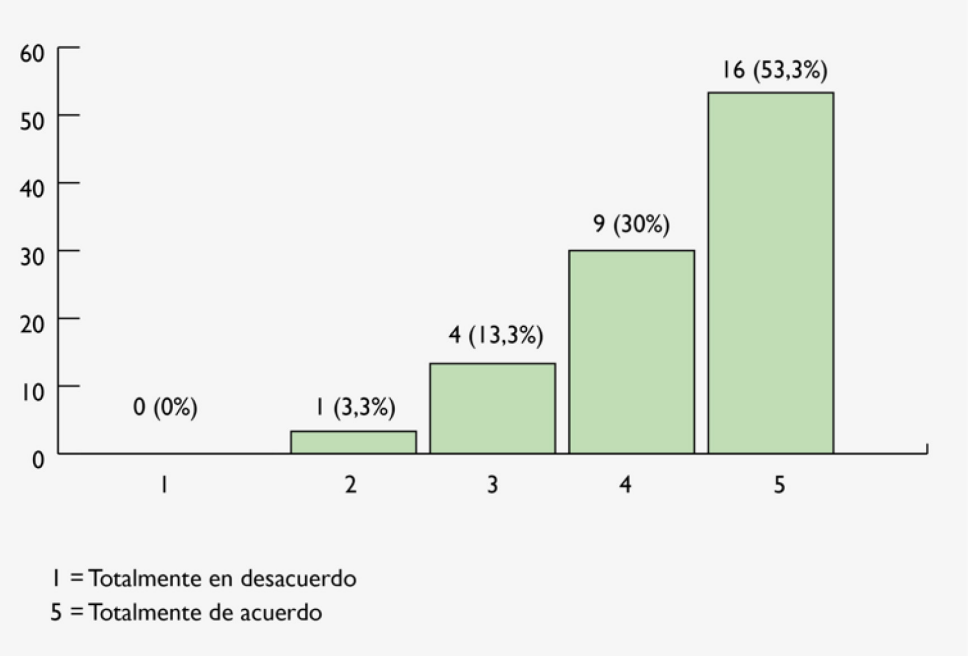

Fig. 7 Resultado de la encuesta a la pregunta: "Disponer de la rúbrica ha ayudado en el aprendizaje” (grupo intervención)

Por el contrario, en el GI la respuesta a la utilidad de la rúbrica (pregunta 8 GI) es mucho más contundente. La suma de los alumnos que están de acuerdo o totalmente de acuerdo en esta pregunta alcanza el 83,3\%, afirmando que disponer de la rúbrica ha sido de gran importancia. Incluso la evaluación de los valores e información que se presenta en la rúbrica (pregunta 9 GI) la consideran más que suficiente, con un $66,7 \%$ con la suma de los valores 4 y 5 (fig. 8 ).

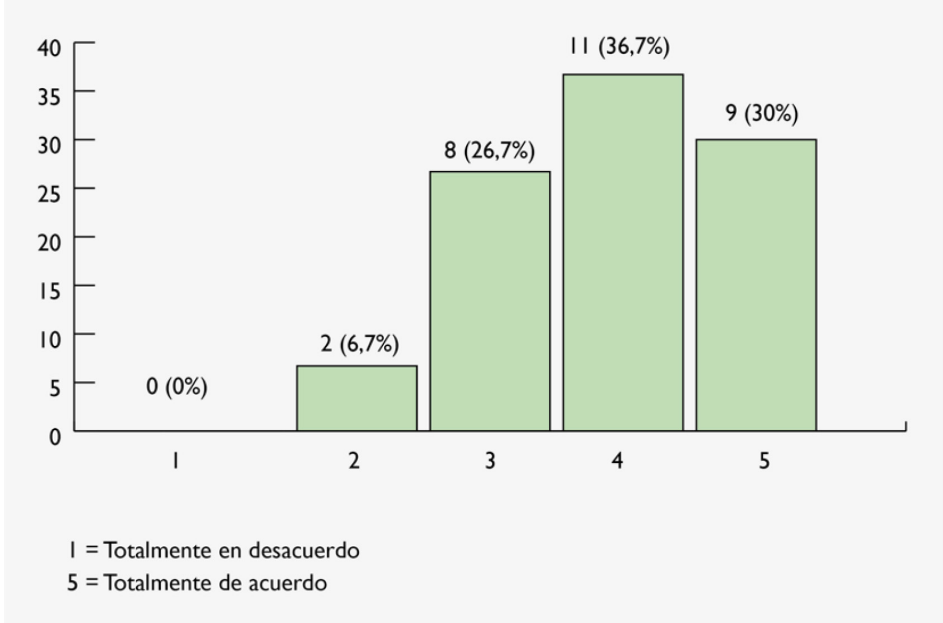

Fig. 8 Resultado de la encuesta a la pregunta 9: "Los valores e información disponible en las rúbricas es suficiente" (grupo intervención)

En la última pregunta planteada a ambos grupos, de respuesta abierta, sobre “¿Qué aspectos crees que se pueden mejorar en la asignatura? Escribe tus propuestas o sugerencias" solo contestaron 12 del GC y 8 
del GI. Los alumnos basan sus propuestas en: realizar más trabajos prácticos en clase y menos explicaciones, disponer de las presentaciones teóricas de las clases y algunos contestan para felicitar a los profesores, indicar que no tienen sugerencias y que esta todo correcto.

Un último resultado de este trabajo se reporta no de la encuesta, sino que viene en relación con las notas medias alcanzadas en los dos últimos años por los grupos que conforman esta asignatura. Se comparan por tanto las notas medias obtenidas antes de la implantación de la innovación (curso 2018/19) y después de la implantación de la innovación (curso 2019/2020). Por un lado, la nota media del GC en estos últimos años se mantiene en un 8,40 , no habiéndose visto una diferencia significativa en el año del ensayo. Pero si nos detenemos en el GI podemos constatar una mejora de la nota media entre curso 2018/19 (nota media; 7,2) y 2019/20 (nota media; 8,10), siendo ésta de casi un punto.

\section{Conclusiones}

La experiencia ha permitido obtener información relevante sobre el uso y valoración que hacen los alumnos de la utilidad para su aprendizaje del material didáctico que se les facilita. Haciendo la muestra con dos grupos de alumnos/as a los que se les ha apoyado de forma distinta para la elaboración de sus trabajos de clase. Los dos grupos, grupo de control (GC) y grupo de intervención (GI), contaban con el mismo número de integrantes y no presentaban diferencias en relación con sus niveles de conocimiento previo en las herramientas informáticas que han aprendido. Estas dos variables, número de componentes por grupo y similares niveles de conocimiento, han permitido construir una encuesta que valore y evalúe la percepción de los alumnos/as en relación con el material complementario disponible.

Los datos mostrados por la encuesta en ambos grupos, tanto el GC como el GI, no reflejan diferencias significativas en ninguna de las preguntas planteadas en el cuestionario. A tenor de los resultados, ambos grupos parece que están satisfechos con el material con el que disponen para abordar los ejercicios de la asignatura. Pero si cuentan con ellos, encuestas en GI, su preferencia se decanta principalmente por el sistema de Lessons en PoliformaT, teniendo muy presentes todos los materiales con los que cuentan en la carpeta de recursos. Así mismo se aprecia diferencia en la demanda de disponer de material didáctico teórico como recurso de la asignatura entre los grupos control frente al grupo intervención que dispone de mayor cantidad de material (Lessons, videoapuntes...). Encajando con la cifra de alumnado del GI que valora positivamente haber contado con este material. Esto ofrece una pista importante sobre la utilidad del material didáctico, como herramienta para el aprendizaje autónomo, ya que su elaboración y uso nos permitirá una mayor disponibilidad de tiempo para los ejercicios prácticos en el horario lectivo en el aula por un lado y permite a los alumnos disponer de un material de consulta para acabar las tareas pendientes en casa.

Por otro lado, la encuesta en ambos grupos refleja un acuerdo en la idoneidad de las herramientas informáticas empeladas, las tareas planteadas y el tiempo empleado para realizarlas. Lo que indica que están conformes con la exigencia (con más o menos dificultad y mucho o poco tiempo empleado) que les supone la realización de las tareas planteadas en la asignatura.

En cuanto al sistema de evaluación, observamos que en ambos grupos hay un elevado grado de satisfacción en el sistema de evaluación planteado de retroalimentación con corrección y re-entrega de las tareas. Encontrando alguna diferencia en relación en el uso de la rúbrica como sistema de evaluación. En este punto queda claro que en contar con ella se convierte en un utensilio importante para la buena ejecución de los ejercicios planteados. 
En la pregunta de respuesta abierta sobre qué aspectos piensa el alumnado que se pueden mejorar en la asignatura, resulta interesante que varios alumnos/as valoran como muy importante el tiempo de trabajo en clase para poder plantear dudas en directo.

El análisis comparativo de los resultados de las encuestas parece indicar que los recursos online de apoyo, así como los sistemas de evaluación que hemos implementado este curso 2019/2020 han sido utilizados y considerados como útiles para la mayoría de los alumnos mostrando un importante grado de satisfacción por parte de los estudiantes que los disponían y un importante grado de interés por tenerlos, por parte de los estudiantes que no disponían de ellos. Sin embargo, debemos centrarnos en mejorar el uso de esos materiales online disponibles para poder así disminuir el tiempo de teoría en el aula y poder ampliar al máximo el tiempo de dedicación a la realización de las tareas prácticas y de aprendizaje del uso de la herramienta en el aula. Esto nos hace replantearnos la programación de las sesiones y la forma en la que se dedica el tiempo en el aula. Actuaciones del tipo docencia inversa donde el alumno debe previamente a las sesiones de clase hacer una serie de tareas en casa podrían ayudar a tener más tiempo disponible en el aula para la realización de las tareas planteadas y la resolución de dudas relativas al uso de las herramientas.

Esta experiencia, gracias a la evaluación obtenida de estos resultados, nos permite reconocer algunos posibles puntos de mejora, aunque somos conscientes de que corresponden a un único cuatrimestre de un curso académico.

Demostrada la mejora en los resultados académicos y en relación con este punto se abre una posibilidad de mejora que pensamos llevar a cabo a lo largo de los próximos años, e involucrando a profesores de otras asignaturas que compartan las mismas estrategias de trabajo. Estrategias que nos podrían ayudar a realizar dinámicas de clase más activas incluso en relación con los sistemas de evaluación, por ejemplo, mediante la preparación de la evaluación por pares para poder alcanzar niveles de aprendizaje de mayor calado, con elementos que generen mayor motivación, mejora en la comprensión de tareas, conceptos y uso de las herramientas.

\section{Referencias}

ARRIBAS, J.M., CARABIAS, D., y MONREAL, I. (2010). "La docencia universitaria en la formación inicial del profesorado. El caso de la escuela de magisterio de Segovia" en Revista Electrónica Interuniversitaria de Formación del Profesorado, 13 (3), p. 27-35. <http://aufop.com/aufop/uploaded_files/articulos/1285861727.pdf> [Consulta: 6 de marzo de 2020]

BLANCO, A. (2008). Las rúbricas: un instrumento útil para la evaluación de competencias en la enseñanza universitaria centrada en el aprendizaje. Barcelona: OCTAEDRO, ICE-UB.

BROWN, J.S., COLLINS, A., y DUGUID, P. (1989). "Situated cognition of learning" en Educational Researcher, 18 , p. 32-42.

CLAUX, M., KANASHIRO, Y., y YOUNG, A. (2001). Modelos Psicológicos de la Instrucción. Lima: Ministerio de Educación.

DÁVILA, S. (2006). “Generación Net: Visiones para su Educación” en ORBIS. 3, p. 24-48.

DÍAZ-BARRIGA, F. (2002). Estrategias docentes para un aprendizaje significativo. Mexico: McGraw-Hill.

FRAILE, A., LÓPEZ-PASTOR, V., CASTEJÓN, J., y ROMERO, R. (2013): "La evaluación formativa en docencia universitaria y el rendimiento académico del alumnado" en Aula Abierta, 41 (2), p. 23-34. 
GESSA, A. (2011). "La coevaluación como metodología complementaria de la evaluación del aprendizaje. Análisis y reflexión en las aulas universitarias" en Revista de Educación, 354, p. 749-764, < http://www.revistaeducacion.mec.es/re354/re354_30.pdf > [Consulta: 6 de marzo de 2020]

HAMODI, C., LÓPEZ V., y LÓPEZ, A.T. (2015). "Medios, técnicas e instrumentos de evaluación formativa y compartida del aprendizaje en educación superior" en Perfiles educativos, 37, p. 146-161.

HAMODI, C., LOPEZ, A.T., Y LOPEZ, V. (2014). "Red de evaluación formativa y compartida en docencia universitaria: creación, consolidación y líneas de trabajo" en Revista de evaluación educativa, 3 (1). < http://revalue.mx/revista/index.php/revalue/article/view/110 > [Consulta: 6 de marzo de 2020]

HERNÁNDEZ, F., FERNÁNDEZ, C., MARTÍNEZ, P., HERVÁS, R. M., y MAQUILLÓN, J. (2002). “Consistencia entre motivos y estrategias de aprendizaje en estudiantes universitarios" en Revista de Investigación Educativa, 20, p. 487-510.

HORTIGÜELA-ALCALA, D., PEREZ-PUEYO, Á., y LOPEZ-PASTOR, V. (2015). “Implicación y regulación del trabajo del alumnado en los sistemas de evaluación formativa en educación superior” en RELIEVE, 21, 1

KLOPPER, H. (2008). “The qualitative research proposal” en Curationis, 31(4) 62-72.

LAURILLARD, D. (1979). “The processes of student learning”. Higher Education, 8, p. 395-409

MARQUÈS, P. (2000). Ventajas e inconvenientes del material multimedia educativo. Departamento de Pedagogía Aplicada, Facultad de Educación, UAB <http://peremarques.net/ventajas.htm> [Consulta: 6 de marzo de 2020].

MARTON, F., y SÄLJO, R. (1976a). “On qualitative differences in learning: I Outcome and process" en British Journal of Educational Psychology, 46, p. 3-11.

MARTON, F., y SÄLJO, R. (1976b). "On qualitative differences in learning: II Outcome as a function of the learner's conception of the task" en British Journal of Educational Psychology, 46, p. 115-127.

McKEACHIE, W. (1986). Teaching Tips: A Guidebook for The Beginning College Teacher (8th Edition). Lexington, MA: D.C. Heath and Co.

MOLTÓ, G. (2012) "Producción y Uso de Vídeo-Ejercicios Didácticos en Asignaturas de Programación” en XVIII Jornadas de Enseñanza Universitaria de la Informática, p. 255-262.

NÚÑEZ, H., CRESPO, E., ÚCAR, X., y BERÑE, A.L. (2014). "Enfoques De Evaluación Orientados A La Participación En Los Procesos De Acción Comunitaria” en Pedagogía social, 24, p. 79-103.

PRENSKY, M. (2001). Digital Natives, Digital Immigrants, en On the Horizon, MCB University Press, 9 (5), $<$ http://www.marcprensky.com/writing/prensky\%20\%20digital\%20natives,\%20digital\%20immigrants \%20\%20part1.pdf> [Consulta: 6 de marzo de 2020]

RODRIGUEZ, G., IBARRA, M.S., y GARCIA, E. (2013) “Autoevaluación, evaluación entre iguales y coevaluación: conceptualización y práctica en las universidades españolas” en Revista de Investigación en Educación, 2 (11), p. 198-210. <http://reined.webs.uvigo.es/ojs/index.php/reined/article/view/708> [Consulta: 6 de marzo de 2020]

SHELDON, T. A. (2005). "Making evidence synthesis more useful for management and policymaking" en $J$ Health Serv Res Policy, $10 \quad$ p. $\quad 1$ p $<$ https://www.researchgate.net/publication/\%207691445_Making_Evidence_Synthesis_More_Useful_for_Manageme nt_and_Policy-Making > [Consulta: 6 de marzo de 2020]

STARKS, H., y TRINIDAD, S. B. (2007). "Choose your method: A comparison of phenomenology, discourse analysis, and grounded theory" en Qualitative health research, 17(10), p. 1372-1380.

UREÑA, N., y RUIZ, E. (2012). "Experiencia de evaluación formativa y compartida en el Máster Universitario en Formación del Profesorado de Educación Secundaria” en Psychology, Society \& Education, 4 (1), p. 29-44 $<$ https://dialnet.unirioja.es/servlet/articulo?codigo=3961254> [Consulta: 6 de marzo de 2020] 
VAN PETEGEM, P., y VANHOOF, J. (2005). "Feedback of performance indicators as a strategic instrument for school improvement" en REICE - Revista Electrónica Iberoamericana sobre Calidad, Eficacia y Cambio en

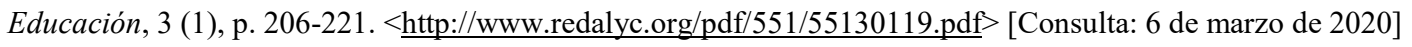

ZOWGHI, D., y SURESH PARYANI. (2003). "Teaching requirements engineering through role playing: Lessons learnt" en Requirements Engineering Conference, 2003. Proceedings. 11 th IEEE International. IEEE, p. 233-241. 